Advanced Computing: An International Journal ( ACIJ ), Vol.3, No.6, November 2012

\title{
A STUDY OF IMAGE COMPRESSION BASED TRANSMISSION ALGORITHM USING SPIHT FOR LOW BIT RATE APPLICATION
}

\author{
Ritu Chourasiya $^{1}$ and Prof. Ajit Shrivastava ${ }^{2}$ \\ ${ }^{(1 \& 2)}$ CSE Deptt. TRUBA, Institute of science \& IT. \\ Bhopal, (M.P.), India. \\ ${ }^{1}$ Chourasiya.ritu@gmail.com \\ ${ }^{2} \mathrm{Ajit} . \mathrm{s} @$ trubainstitute.ac.in
}

\begin{abstract}
Image compression is internationally recognized up to the minute tools for decrease the communication bandwidth and save the transmitting power. It should reproduce a good quality image after compressed at low bit rates. Set partitioning in hierarchical trees (SPIHT) is wavelet based computationally very fast and among the best image compression based transmission algorithm that offers good compression ratios, fast execution time and good image quality. Precise Rate Control (PRC) is the distinct characteristic of SPIHT. Image compression-based on Precise Rate Control and fast coding time are principally analyzed in this paper. Experimental result shows that, in the case of low bit-rate, the modified algorithm with fast Coding Time and Precise Rate Control can reduce the execution time and improves the quality of reconstructed image in both PSNR and perceptual when compare to at the same low bit rate.
\end{abstract}

\section{KEYWORDS}

Wavelet analysis, image compression, SPIHT algorithm, Precise Rate Control (PRC).

\section{INTRODUCTION}

Digital image compression is now essential. Internet teleconferencing, High Definition Television (HDTV), satellite communications and digital storage of images will not be feasible without a high degree of compression. Wavelets [1] became popular in past few years in mathematics and digital signal processing area because of their ability to effectively represent and analyze data. Typical application of wavelets in digital signal processing is image compression. Image compression algorithms based on Discrete Wavelet Transform (DWT)[2],such as Embedded Zero Wavelet (EZW)[3] which produces excellent compression performance, both in terms of statistical peak signal to noise ratio (PSNR) and subjective human perception of the reconstructed image[10][11]. Said and Pearlman [4] further enhanced the performance of EZW by presenting a more efficient and faster implementation called set partitioning in hierarchical trees. SPIHT is one of the best algorithms in terms of the peak signal-to-noise ratio (PSNR) and execution time. Set partitioning in hierarchical trees [5] provide excellent rate distortion performance with low encoding complexity.

It is clear that traditional image compression techniques like JPEG and MPEG produce 
annoying visual degradation when operating at low bit rates because they introduce errors in visually important parts of the image structure and also introduces the 'blocking artifacts' in the reproduced images [1][2][3]. Since compressed images are to be transmitted over the data communication network or a wireless network, where the bandwidth of the link cannot be guaranteed. Therefore the rate scalable image compression methods are appealing for low bit rate applications, as the application of battery based devices is increasing rapidly in image capturing, storing and transmission over web based networks.

There is a need of efficient image compression method that must be suitable for the data communication networks. On the same hand, compression method should be suitable for the devices having limited battery life and on board memory. The main objective of this paper is to propose an image compression and transmission algorithm which is suitable for low bit rate applications over Internet or any other [6][7]wireless network.

It needs to maintain a perfect balance between available bandwidth and perceived quality of received image, with minimum transmission delays .Important factors to be considered are compression time, algorithm complexity, cost and computational resources. Considering such factors, one requires a compression method which should be simple and fast. It should reproduce a good quality image after compression at low bit rates.

The rest of the paper is organized in following sections. In section-II Original SPIHT and its steps, In section-III Proposed method, In section-IV Experimental result, In section-V Conclusion, Lastly, paper concludes with References.

\section{ORIGNAL SPIHT}

Set Partitioning in Hierarchical Trees (SPIHT) algorithm is based on embedded zero tree wavelet (EZW) coding method; it employs spatial orientation trees and uses set partitioning sorting algorithm [5][7]. Coefficients corresponding to the same spatial location in different sub bands in the pyramid structure display self-similarity characteristics. SPIHT defines parentchildren relationships between these self- similar sub bands to establish spatial orientation trees.

\subsection{Steps in SPIHT Algorithm}

Step1: In the sorting pass, the List of Insignificant Pixel (LIP) is scanned to determine whether an entry is significant at the current threshold. If an entry is found to be significant, output a bit ' 1 ' and another bit for the sign of the coefficient, which is marked by either ' 1 ' for positive or ' 0 ' for negative. Now the significant entry is moved to the list of significant pixel (LSP). If an entry in LIP is insignificant, a bit ' 0 ' is output.

Step2: Entries in List of Insignificant Set (LIS) are processed. When an entry is the set of all descendants of a coefficient, named 'type A', magnitude tests for all descendants of the current entry are carried out to decide whether they are significant or not. If the entry is found to be as significant, the direct offspring's of the entry undergoes magnitude tests. If direct offspring is significant, it is moved into LIP; otherwise it is moved into LSP. If the entry is deemed to be insignificant, this spatial orientation tree rooted by the current entry was a zero-tree, so a bit ' 0 ' is output and no further processing is needed. 
Finally, this entry is moved to the end of LIS as 'type B', which is the set of all descendants except for the immediate offspring of a coefficient. If the entry in LIS is type B, significance test is performed on the descendants of its direct offspring. If significance test is true, the spatial orientation tree with root of type B entry is split into four sub-trees that are rooted by the direct offspring and these direct offspring's are added in the end of LIS as type A entries. The important thing in LIS sorting is that entire sets of insignificant coefficients, zero-trees, are represented with a single zero. The purpose behind defining spatial parent-children relationships is to increase the possibility of finding these zero-trees.

Step3: Finally, refinement pass is used to output the refinement bits ( ${ }^{\text {th }}$ bit) of the coefficients in LSP at current threshold. Before the algorithm proceeds to the next round, the current threshold is halved.

\section{OPTIMIZATION OF SPIHT ALGORITHM}

The proposed SPIHT algorithm is a fast and efficient technique for image compression and transmission at lower bit rates over [6] any network. SPIHT algorithm mainly depends upon its three lists vise LIP, LIS and LSP.

$>$ List of Insignificant Pixels (LIP): The list of insignificant pixels (LIP) contains individual coefficients that have magnitudes smaller than the threshold. This list keeps track of pixels to be evaluated.

$>$ List of Insignificant Set (LIS): The list of insignificant sets (LIS) contains sets of wavelet coefficients that are defined by tree structures and are found to have magnitudes smaller than the threshold (insignificant). The sets exclude the coefficients corresponding to the tree and all sub tree roots and they have at least four elements. This list is one that shows us that we are saving work by not accounting for all coordinates but just the relative ones.

$>$ List of Significant Pixels (LSP): The list of significant pixels (LSP) is a list of pixels found to have magnitudes larger than the threshold (significant). This list keeps track of pixels already evaluated and need not be evaluated again.

It is the characteristic of SPIHT algorithm that it generally operates on an entire image at once. Due to this the size of the three lists is often quite big and takes a lot of memory. The whole image is loaded and transformed, and then the algorithm requires repeated access to all lists. This consumes much time in encoding and compressing the image[8][12]. This is the disadvantage of the SPIHT algorithm for the small mobile devices having limited memory and processing capability when they are compressing an image for transmission over any network.

Secondly, at lower bit rates, maintaining the precise rate control is essential condition for both encoding and decoding of the image. Precise rate control mechanism is one of the features of the proposed work. Therefore, in this proposed work, the SPIHT algorithm has been modified to with the following two constraints.

- Fast Coding Time

- Precise Rate Control 


\subsection{Fast Coding Time}

The computation time mainly consists of two components.

In applying the wavelet transform on the image.

$>$ Time consumed in compressing the image.

In this paper, time consumed in compressing the image is focused. The modification lies in the working and utility of the LSP. As per the modifications done in algorithm, the LSP list is completely eliminated. This means that now there is no need to access the list in refinement pass. The LSP list contains thousands of elements and each element of the list is to be access every time to output most significant bit. This consumes much time. After modification the LSP list is completely eliminated therefore time required to access each element of LSP is saved. Thus reduces the encoding time considerably without affecting the quality of reproduced image much.

\subsection{Precise Rate Control}

Due to the modifications in the algorithm, a number of bits are outputted at once instead of one bit per one significant pixel value. This modification disturbs the precise rate characteristics of the SPIHT algorithm. Maintaining the precise rate control is necessary at low bit rates [2][3]. Precise rate control means a mechanism to truncate the encoding and decoding as soon as the allocated bit budget is fully utilized. Therefore an effective precise rate mechanism is also implemented along with optimized SPIHT.

\subsubsection{Bit Budget}

Bit budget is defined as the number of bits required to represent a compressed image at a given bit rate. It can be defined as the bit bank, which issues the bit or bits to particular pixel or value. In compression, the effective utilization of the bit budget is very necessary for the quality reproduction of the given bit rate. It is necessary that significant information should get the maximum number of bits and the least significant information should get the least number of bits. The mechanism of the SPIHT algorithm is well suited for the effective utilization of the allocated bit budget. This mechanism is maintained while modifying the SPIHT algorithm.

\subsubsection{Calculation of Bit Budget}

Let the required bit rate be 'B'.

Let the size of picture be $\mathbf{M} \mathbf{x} \mathbf{N}$

Let the bit depth be ' $\mathbf{b}$ ' bits per pixel

Then the total bits $=\mathbf{M} \mathbf{x} \mathbf{N} \mathbf{x} \mathbf{b}$

And, Bit Budget $=\mathbf{B} \mathbf{x} \mathbf{M} \mathbf{x} \mathbf{N} \mathbf{x}$ b bits 
Advanced Computing: An International Journal ( ACIJ ), Vol.3, No.6, November 2012

According to the above formula, the Table 1 shows the bit budget calculated at different bit rates. The table clearly shows that as the bit rate increases, the bit budget also increases. It also shows that bit rate and compression ratios are different parameters. Bit rate is independent of the bit depth but compression ratio depends upon the bit depth also.

Table 1.Calculation of Bit Budget

\begin{tabular}{|c|c|c|c|c|}
\hline S.no & Size of image & Bit depth (bpp) & Bit rate (bpp) & Bit budget (bits) \\
\hline 1. & $\mathbf{5 1 2} * \mathbf{5 1 2}$ & $\mathbf{8}$ & $\mathbf{0 . 1}$ & $\mathbf{2 6 2 1 4}$ \\
\hline 2. & $\mathbf{5 1 2} * \mathbf{5 1 2}$ & $\mathbf{8}$ & $\mathbf{0 . 2}$ & $\mathbf{5 2 4 2 9}$ \\
\hline 3. & $\mathbf{5 1 2} * \mathbf{5 1 2}$ & $\mathbf{8}$ & $\mathbf{0 . 3}$ & $\mathbf{7 8 6 4 3}$ \\
\hline 4. & $\mathbf{5 1 2} * \mathbf{5 1 2}$ & $\mathbf{8}$ & $\mathbf{0 . 4}$ & $\mathbf{1 0 4 8 5 8}$ \\
\hline 5. & $\mathbf{5 1 2} * \mathbf{5 1 2}$ & $\mathbf{8}$ & $\mathbf{0 . 5}$ & $\mathbf{1 3 1 0 7 2}$ \\
\hline $\mathbf{6 .}$ & $\mathbf{5 1 2} * \mathbf{5 1 2}$ & $\mathbf{8}$ & $\mathbf{0 . 6}$ & $\mathbf{1 5 7 2 8 6}$ \\
\hline 7. & $\mathbf{5 1 2} * \mathbf{5 1 2}$ & $\mathbf{8}$ & $\mathbf{0 . 7}$ & $\mathbf{1 8 3 5 0 1}$ \\
\hline
\end{tabular}

\section{EXPERIMENTAL RESULT}

The proposed optimized image codec algorithm has applied on several images at different bit rate and we have compared the result (coding time and quality) of our proposed method with other image compression methods.

The Table 2 shows the reduction in time of execution using optimized algorithm as compared to original SPIHT.

Table 2.Coding Time Test

\begin{tabular}{|c|c|c|c|}
\hline S.no & $\begin{array}{c}\text { Bit rate } \\
\text { (bpp) }\end{array}$ & $\begin{array}{c}\text { Time consumed in } \\
\text { SPIHT(sec) }\end{array}$ & $\begin{array}{c}\text { Time consumed in optimized } \\
\text { SPIHT(sec) }\end{array}$ \\
\hline 1. & $\mathbf{0 . 1}$ & $\mathbf{2 . 2 6 0 6 7 9}$ & $\mathbf{1 . 8 4 9 6 2 5}$ \\
\hline 2. & $\mathbf{0 . 2}$ & $\mathbf{5 . 2 7 6 2 1 5}$ & $\mathbf{4 . 7 0 6 8 9 7}$ \\
\hline 3. & $\mathbf{0 . 3}$ & $\mathbf{1 0 . 5 7 1 7 6 7}$ & $\mathbf{8 . 9 5 1 6 8 8}$ \\
\hline 4. & $\mathbf{0 . 4}$ & $\mathbf{1 4 . 2 5 0 8 1 2}$ & $\mathbf{1 3 . 8 4 7 0 4 2}$ \\
\hline 5. & $\mathbf{0 . 5}$ & $\mathbf{2 1 . 7 3 9 1 2 4}$ & $\mathbf{1 6 . 5 1 9 0 2 2}$ \\
\hline 6. & $\mathbf{0 . 6}$ & $\mathbf{3 4 . 1 2 7 2 9 3}$ & $\mathbf{2 1 . 3 4 8 7 7 8}$ \\
\hline
\end{tabular}

In Figure1. Shows that the less time consumed by proposed method at same bit rate the original SPIHT method takes large time for coding.

To evaluate the effectiveness of our proposed method, we have used the transmission time (Coding time) and PSNR.

By comparing the transmission time and PSNR of other methods such as JPEG, JPEG 2000 and SPIHT with proposed method inFigure2. 


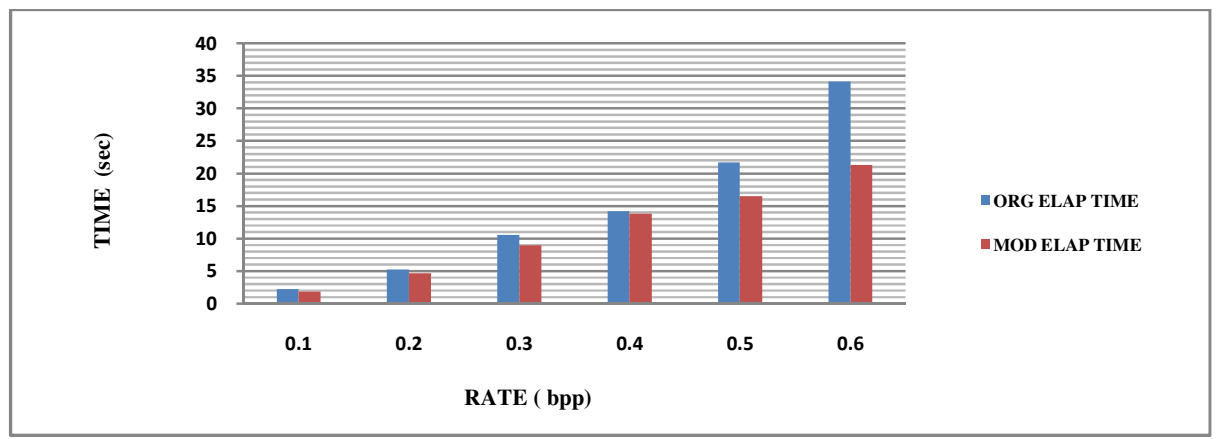

Figure 1.Coding time comparison at various bit rates

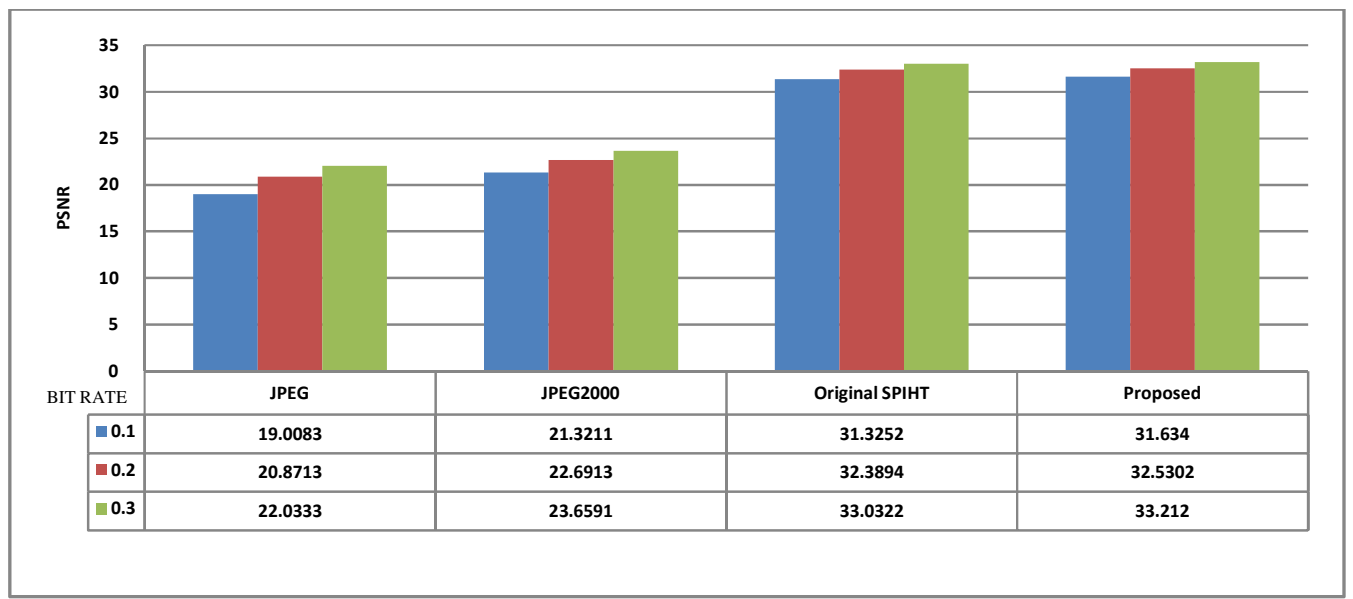

Figure 2. Comparison of different image code quality (in PSNR)

PSNR is use to measure the quality of reconstructed images that have been compressed. When an image is compressed and reconstructed the value of each picture element can be change.

$\operatorname{PSNR}(d B)=10 \log 10^{\left(\frac{255^{2}}{M S E}\right)}$

Signals can have a wide dynamic range, so PSNR is usually expressed in decibels, which is a logarithmic scale. It shows that a higher PSNR value provides a higher image quality.

Table3.Image Quality

\begin{tabular}{|c|c|c|}
\hline \multirow{2}{*}{ METHODS } & \multicolumn{2}{|c|}{ Leena image at 0.1bit rate } \\
\cline { 2 - 3 } & PSNR & Coding time \\
\hline JPEG & 19.0083 & $\mathbf{8 . 4 5 6 8 0 3}$ \\
\hline JPEG2000 & 21.3211 & 7.235924 \\
\hline Original SPIHT & 31.3770 & 2.306803 \\
\hline Proposed & 31.7541 & 2.095924 \\
\hline
\end{tabular}




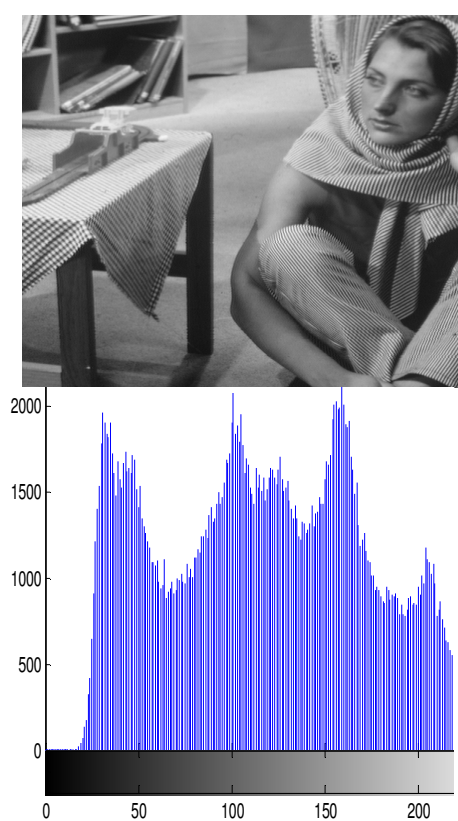

(a)

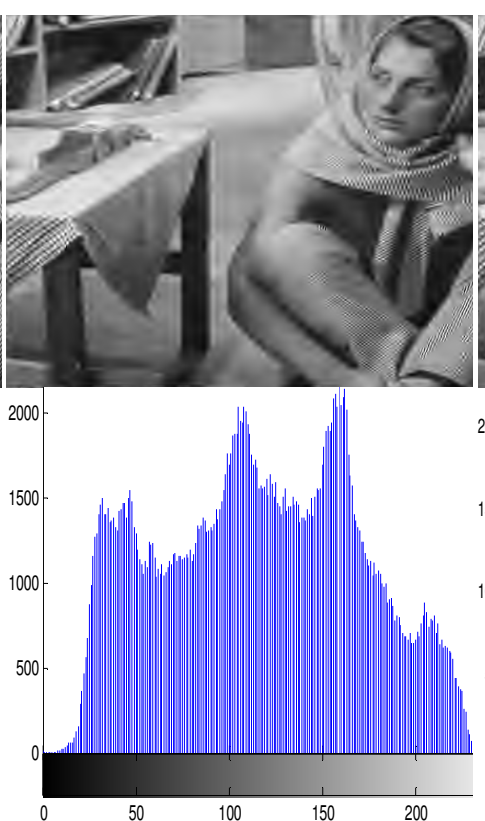

(b)

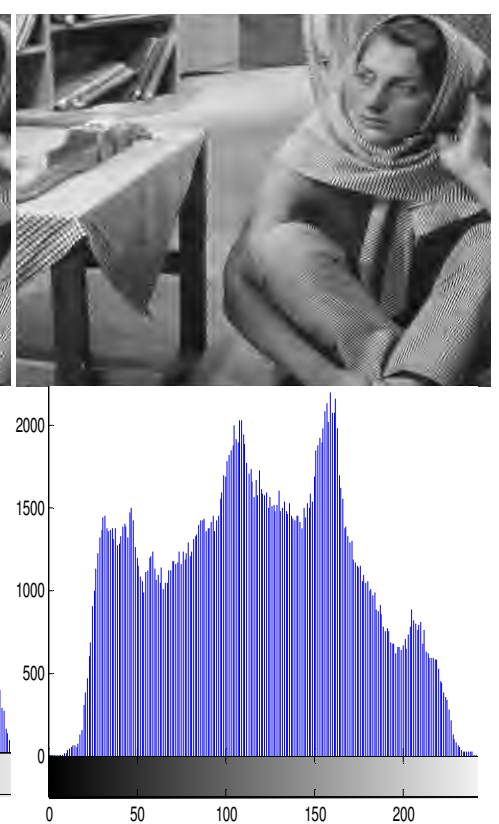

(c)

Figure 3.Show Leena image coded with corresponding PSNR and Elapsed time with its histogram at 0.1 bit rate

(a) Original Image (b) Image Reconstructed, SPIHT, PSNR=31.3770db, ELAP Time 2.306803sec. (c) Image Reconstructed, using Modified SPIHT, PSNR=31.7541db, ELAP Time 2.095924sec.

The Mean Square Error (MSE) is shows the difference (cumulative squared error) between the original and the compressed image.

$M S E-\frac{1}{m n} \sum_{i=0}^{m-1} \sum_{j=0}^{n-1}[I(i, j)-K(i, j)]^{2}$

WhereI $(i, j)$ is the original image, $\mathrm{K}(\mathrm{i}, \mathrm{j})$ is the decompressed image and $\mathrm{m}, \mathrm{n}$ are the dimensions of the images. A lower value for MSE means lesser error.

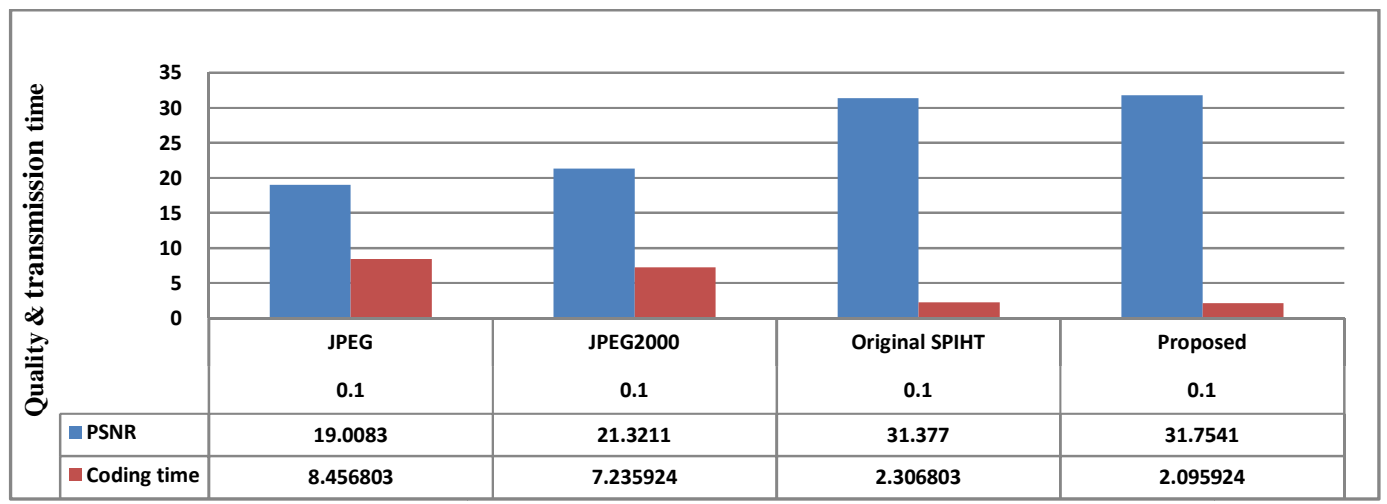

Figure 4. Comparison of different method code at 0.1 bit rate 


\section{CONCLUSION}

In this paper, we proposed an efficient and fast compression based transmission method which is suitable for the transmission and compression of images over data communication network. It maintaining a perfect balance between available bandwidth and perceived quality of received image, with minimum transmission delays .The algorithm gives sufficient compression and a good visual and objective quality of the reconstructed image.

The comparison of coding time and PSNR of optimized method with simple SPIHT method clearly shown above that the proposed method reduces the time to encode image and maintain better PSNR at low bit rate. In this paper we achieved fast transmission at low bit rate without degrade the quality of the image.

\section{REFERENCES}

[1] Meng Wang and Qi Han, "An Improved Algorithm of SPIHT based on Human Visual Characteristics", International Journal of Biomedical Science Vol.1, Pg. No. 423-335 2005.

[2] Chang Shoarang and Lawrence Carin, "A modified SPIHT algorithm for image coding with a joint MSE and classification distortion measure" IEEE Transaction on Image Processing, Vol 15 Pg. No. 713-725, March 2006.

[3] Said Amir and Pearlman William A., "A New Fast and Efficient Image Codec Based on Set Partitioning in Hierarchical Trees” ,IEEE Transactions On Circuits and Systems For Video Technology, Vol.6, Pg. No. 243-250, June 1996.

[4] Jianxiong Wang and Fuxia Zhang "Study of the Image Compression based on SPIHT Algorithm" International Conference on Intelligent Computing and Cognitive Informatics Vol.3, Pg. No. 131-133 2010 IEEE.

[5] Cebrail Taskin and Serdar Kürşat Sarikoz "An Overview of Image Compression Approaches "The Third International Conference on Digital Telecommunications Vol.4, Pg. No. 174-179 2008 IEEE.

[6] Sonja Grgic, Marta Mrak, Mislav Grgic, " Comparison of JPEG image coder", Proceedings of the 3rd International Symposium on Video Processing Pg. No. 217-223, 2005.

[7] Weixing Zhang and Thomas R. Fischer, "Comparison of Different Image Subband Coding Methods at Low Bit Rates” IEEE Transaction on Image Processing, VOL. 9, APRIL 1993.

[8] Todd Owen, Scott Hauck “Arithmetic Compression on SPIHT Encoded Images" International Conference on Intelligent Computing and Cognitive Informatics Vol.3, Pg. No. 131-133 2010 IEEE.

[9] Said Amir and Peralman, "Image Compression Using The Spatial Orientation Tree", IEEE 1993.

[10] Lewis A.S. and Knowles G., "Image compression Using the 2-D wavelet Transform", IEEE Transactions on Image Processing, Vol. 1 No. 2 Pg. No. 244-250 April 1992.

[11] Sonja Grgic, Mislav Grgic, Yuen Cheng, "Performance Analysis of Image Compression using Wavelets",IEEE transaction on Industrial Electronics, Vol. 48, No. 3 , Pg. No. 682-695, June 2001.

[12] Yong Sun, Hui Zhang, "Real time Implementation of New Memory SPIHT Image Coding Algorithm Using DSP Chip" ,IEEE Transaction On Image Processing, Vol. 11, No.9, September 2002. 